than three times that removed by the tooth flanks and that the volume of metal removed by a tip is almost twice that removed by one flank, thus explaining the fact experienced in practice, that the loading of the tips of the hob teeth is greater than that of the flanks. They also established that the volumes of material removed by the two flanks of one tooth space are unequal and that the maximum loadings on the tip and flanks do not occur simultaneously. This not only results in fluctuating machining forces but may also lead to a reversing torque acting on the table which carries the workpiece, and this can be dangerous if excessive backlash exists in the table drive.

The results of the computer calculations apply, of course, only to the particular example for which they were carried out, but the principle will be applicable to other gear geometries and will thus be of general interest and value.

\section{Cephalopod Catalogue}

The first in a series listing invertebrate fossil type specimens of the Division of Invertebrate Paleontology of the Smithsonian Institution has just been published (US National Museum Bulletin 262, part 1; 1968). It covers the collection of Palaeozoic Cephalopoda, and it has been compiled by Louis R. Purnell, a member of the museum staff. The only other published catalogue (USNM Bulletin 53, part 1; 1905) covered the complete collection of fossil invertebrates held at that time. Since then, the type collections have grown from approximately 11,500 to nearly 200,000 . Included in this figure are some $\mathbf{5 5 , 4 0 0}$ small foraminifera, 25,000 brachiopods, 9,000 crinoids and 12,300 trilobites. The Palaeozoic cephalopods in the collection today number some 4,250.

The cephalopod catalogue is split into four chief chapters: Nautiloidea, Ammonoidea, Bactritoidea and Coleoidea. Within each chapter the taxa are listed alphabetically by genus and species. Individual citations include the binomen, author, date, page, plate, figure number, kind of type, catalogue number, rock unit as originally designated, and locality. The catalogue sets out to be only a brief listing of type specimens, and therefore it is not a source book for occurrence data, synonymies or stratigraphic interpretation of any kind.

\section{Proteins Sequenced}

THE current edition of the Atlas of Protein Sequence and Structure, published annually by the National Biomedical Research Foundation, lists the amino-acid sequences of more than 200 proteins. This is double the number that appeared in last year's edition and constitutes a signal tribute to the industry of biochemists as well as an indication of the interest that is aroused by the subject.

The protein molecules sequenced, in whole or in part, include 36 cytochrome- $C$ s, 5 ferrodoxins, 20 alpha and 15 beta haemoglobins, 4 myoglobins, 12 Bence-Jones proteins, 4 ribonucleases and 22 othor assorted enzymes. There are more than 30 pituitary hormones, glucagon, secretin, 18 insulin $A$ chains and 19 insulin $B$. Five transfer-RNA sequences have also been included.

The text of the Atlas lists amino-acid residues by the familiar three letter symbols as well as by the single letter symbols recommended by the Commission on Biochemical Nomenclature. The alignments of homologous sequences, printed on fold-out sheets at the back of the Atlas, employ the single letter symbols alone and are thereby enabled to display entire sequences on a single line.

The Atlas is far from being a mere catalogue of other people's work; it includes a considerable amount of discussion and statistical analyses of the data it contains. Where appropriate, node point sequences in phylogenetic trees have been worked out by computer and are tabulated with the sequence alignments.

Many proteins of known sequence have been determined because of their medical or biochemical interest and their significance for evolutionary studies has been of perhaps secondary importance in the eyes of their investigators. There is thus a certain disparity in the distribution of sequence data among biological groups. In the mammalian kingdom 146 protein sequences of more than 30 residues have been determined, whereas insects and plants boast only three proteins each. No proteins of this length or greater have been sequenced for molluscs, protozoa or algae. The selection of significant proteins for sequencing will depend on careful judgment and doubtless a considerable measure of luck.

The Atlas is edited by Margaret $O$. Dayhoff and Richard V. Eck and is available from the National Biomedical Research Foundation at the trifling price of $\$ 6$.

\section{Enzymes and NMR}

\section{from our Molecular Biology Correspondent}

ONE of the most noticeable trends in the past year or so in the field of structure and mechanism of enzymes has been the increasingly widespread use of nuclear magnetic resonance to study conformation and binding. Much of this work still represents, as it were, a flexing of muscles, involving, as it does, the examination of systems of known three-dimensional structure, which can therefore be used to practise assignment and interpretation of frequencies. In principle, NMR spectra of the smaller enzymes may be expected to produce new data on the composition and geometry of active sites, the types of interactions with the substrate and even on the kinetic parameters of the system.

The identification of resonances in the lysozyme spectrum has been extended by Cohen and Jardetzky (Proc. US Nat. Acad. Sci., 60, 92; 1968), who have examined the part of the spectrum (low-field) arising from the aromatic residues, which are abundant in this protein. Compared with the fully denatured enzyme, the native molecule shows certain characteristic shifts, which allow several assignments to be made. Some interactions, in particular that between the two adjacent tryptophans 62 and 63 , are very tenacious and are not eliminated even in $8 \mathrm{M}$ urea. The most interesting effects, however, concern binding of the specific inhibitors, $N$-acetylglucosamine and its dimer and trimer. The monomer shows only a small effect, but the dimer and trimer, which bind strongly, produce some striking changes. As the inhibitor is added, a new peak appears in the spectrum, which shifts downfield as saturation is approached. 'This is similar to an 